\title{
Imaging of Leukocyte Trafficking in Alzheimer's Disease
}

\author{
Enrica Pietronigro, Elena Zenaro and Gabriela Constantin* \\ Section of General Pathology, Department of Medicine, University of Verona, Verona, Italy
}

Alzheimer's disease (AD) is the most common neurodegenerative disorder and is characterized by a progressive decline of cognitive functions. The neuropathological features of $A D$ include amyloid beta $(A \beta)$ deposition, intracellular neurofibrillary tangles derived from the cytoskeletal hyperphosphorylated tau protein, amyloid angiopathy, the loss of synapses, and neuronal degeneration. In the last decade, inflammation has emerged as a key feature of $A D$, but most studies have focused on the role of microglia-driven neuroinflammation mechanisms. A dysfunctional blood-brain barrier has also been implicated in the pathogenesis of $A D$, and several studies have demonstrated that the vascular deposition of $A \beta$ induces the expression of adhesion molecules and alters the expression of tight junction proteins, potentially facilitating the transmigration of circulating leukocytes. Two-photon laser scanning microscopy (TPLSM) has become an indispensable tool to dissect the molecular mechanisms controlling leukocyte trafficking in the central nervous system (CNS). Recent TPLSM studies have shown that vascular deposition of $A \beta$ in the CNS promotes intraluminal neutrophil adhesion and crawling on the brain endothelium and also that neutrophils extravasate in the parenchyma preferentially in areas with $A \beta$ deposits. These studies have also highlighted a role for LFA-1 integrin in neutrophil accumulation in the CNS of AD-like disease models, revealing that LFA-1 inhibition reduces the corresponding cognitive deficit and AD neuropathology. In this article, we consider how current imaging techniques can help to unravel new inflammation mechanisms in the pathogenesis of $A D$ and identify novel therapeutic strategies to treat the disease by interfering with leukocyte trafficking mechanisms.

Keywords: Alzheimer's disease, leukocyte trafficking, two-photon laser scanning microscopy

Specialty section:

This article was submitted to Multiple Sclerosis and Neuroimmunology,

a section of the journal

Frontiers in Immunology

Received: 30 November 2015

Accepted: 23 January 2016

Published: 15 February 2016

Citation:

Pietronigro E, Zenaro E and Constantin G (2016) Imaging of

Leukocyte Trafficking in

Alzheimer's Disease.

Front. Immunol. 7:33.

doi: 10.3389/fimmu.2016.00033

\section{INTRODUCTION}

Alzheimer's disease (AD) is the most common neurodegenerative cause of dementia in the elderly and is characterized by a progressive deterioration of cognitive functions. The neuropathological features include amyloid beta (A $\beta$ ) neuritic plaques, neurofibrillary tangles (NFTs) comprising aggregates of hyperphosphorylated microtubule tau protein, amyloid angiopathy, and the loss of neurons and synapses (1). The major pathogenic concept in the field of AD research is the amyloid cascade hypothesis, which states that the sequence of pathological events leading to $\mathrm{AD}$ is characterized by the accumulation of $A \beta$ peptides resulting from the aberrant processing of amyloid precursor protein (APP) and dysfunctional A $\beta$ clearance, followed by the deposition of NFTs and the onset of synaptic dysfunction and neuronal loss. A $\beta$ peptides are formed by the proteolytic cleavage of APP due to the sequential activities of $\beta$-site APP-cleaving enzyme 1 (BACE-1) ( $\beta$-secretase), $\gamma$-secretase, 
and a protein complex with presenilin 1 (PS1) at its catalytic core. Although amyloid plaques and aggregated tau are both part of the neuropathological definition of the disease, numerous studies suggest that soluble oligomeric forms of $A \beta$ and tau are the predominant mediators of cytotoxicity in $\mathrm{AD}(2)$.

Alzheimer's disease pathology is also characterized by an inflammatory response primarily driven by cytokines and the intrinsic myeloid cells in the brain, which are known as microglia (3). It is now widely accepted that microglia-mediated neuroinflammatory responses may promote the neurodegeneration observed in $\mathrm{AD}(1,3)$. Microglial activation precedes neuropil loss in $\mathrm{AD}$ patients, and recent genome-wide association studies have revealed that microglial genes, such as CD33, TREM2, and $H L A-D R$, are associated with susceptibility to the late-onset form of the disease (3). Furthermore, in response to A $\beta$ or NFTs, microglial cells produce proinflammatory cytokines, chemokines, and complement peptides, which can recruit leukocyte subpopulations to the brain. $A \beta$ also stimulates microglia to produce reactive nitrogen intermediates, such as nitric oxide (NO) and reactive oxygen species (ROS), and the resulting oxidative stress induces neuronal damage (3).

Circulating leukocytes may also play a role in the inflammation process during $\mathrm{AD}$. The migration of leukocytes from blood vessels into the central nervous system (CNS) involves a sequence of adhesion and activation events including (1) capture (tethering) and rolling, which are mediated by the interactions between selectins and mucins, and/or between integrins and proteins with immunoglobulin domains; (2) activation induced by chemokines, resulting in the subsequent activation of integrins; (3) arrest mediated by integrins and their counter-ligands; and (4) diapedesis or transmigration (4). Additional steps may include slow rolling, adhesion strengthening and spreading, and intravascular crawling (5). Leukocyte trafficking in the CNS during inflammatory diseases is mediated predominantly by endothelial E-selectin, P-selectin, and their mucin ligands, as well as leukocyte integrins including $\alpha 4 \beta 1$ (also known as very late antigen 4 , VLA-4) and $\alpha \mathrm{L} \beta 2$ (also known as leukocyte functionassociated antigen 1, LFA-1), which bind the endothelial vascular cell adhesion molecule (VCAM-1) and intercellular cell adhesion molecule (ICAM-1), respectively.

The role of circulating immune system cells in AD-related brain damage is poorly understood, but the use of in vivo imaging techniques, such as two-photon laser scanning microscopy (TPLSM), can provide insights into the mechanisms controlling leukocyte trafficking in $\mathrm{AD}$ and may lead to the development of novel therapeutic strategies to delay the progression of the disease. In this review, we discuss recent work on the role of circulating leukocytes in $\mathrm{AD}$, highlighting the use of in vivo imaging to investigate leukocyte recruitment in the CNS and to study the basis of novel disease mechanisms.

\section{LEUKOCYTE TRAFFICKING IN AD}

Monocytes are circulating leukocytes that play an important role in the innate immune response against pathogens. Numerous studies have shown that peripheral myeloid cells can infiltrate brain tissue and reduce the deposition of $A \beta$ plaques (6-9).
The entry of monocytes into the CNS is tightly regulated and involves the CC-chemokine ligand 2 (CCL2)-CCR2 axis (10). $\mathrm{A} \beta$ is chemotactic for monocytes, and it induces the secretion of proinflammatory cytokines and monocyte transendothelial migration in a blood-brain barrier (BBB) model, in a process that involves the $\mathrm{A} \beta$ receptor (RAGE) and platelet endothelial cell adhesion molecule (PECAM-1) expressed on endothelial cells $(11,12)$. In agreement with these in vitro studies, the injection of synthetic A $\beta$ peptides into the hippocampus triggers the trafficking of bone-marrow-derived monocytic cells into the brain, which then differentiate into ramified microglia and penetrate into the core of $A \beta$ plaques $(7,13)$. Furthermore, recent data indicate that infiltrating monocytes rather than resident microglia express TREM2, a receptor involved in myeloid cell phagocytosis, further supporting the role of peripheral myeloid cells in AD pathogenesis (14). Intravital TPLSM has elegantly confirmed that patrolling monocytes are attracted to and crawl onto the luminal walls of $A \beta$-positive veins but not $A \beta$-positive arteries or A $\beta$-negative blood vessels (15).

Neutrophils are the most abundant population of cells in the blood and are the primary mediators of the innate immune response. Previous reports $(16,17)$, including our own results (18), have shown that neutrophils do not necessarily need to accumulate in tissues in high numbers in order to induce tissue damage: intravascular adhesion per se without transmigration is sufficient to induce endothelial injury. The role of neutrophils in the induction of neuropathological changes and memory deficit in AD models has been demonstrated only recently (19). A higher number of infiltrating neutrophils was observed at the onset of cognitive deficits in 5xFAD and 3xTg-AD mice, especially in the cortex and hippocampus. In corresponding TPLSM studies, neutrophil extravasation was observed at the early stage of the disease inside the cerebral parenchyma, particularly in areas adjacent to vascular $A \beta$ deposits or rich in intraparenchymal $A \beta$ plaques (19). Similarly, Gr1+-labeled cells also infiltrate the brain parenchyma of $5 x F A D$ mice and migrate toward $\mathrm{A} \beta$ plaques $(20)$. These data, together with previous in vitro results, suggest that $A \beta$ plays a role in the chemotaxis and accumulation of neutrophils in the brains of $\mathrm{AD}$ mice $(21,22)$. Furthermore, soluble $\mathrm{A} \beta$ oligomers rapidly trigger neutrophil adhesion to integrin ligands in vitro and induce the transition of LFA-1 integrin from the low- to the high-affinity binding state, suggesting that $\mathrm{A} \beta$ plays a key role in neutrophil intravascular adhesion and migration into the brain during AD (19). Neutrophils migrate into the brains of mice with AD-like disease by using LFA-1 integrin, which controls both intravascular adhesion and intraparenchymal motility. Indeed, the treatment of $\mathrm{AD}$-like transgenic mice with monoclonal antibodies that block LFA-1 integrin or deplete neutrophils at early stages of the disease (when mice start to present memory impairment) mitigated the neuropathological hallmarks of $\mathrm{AD}$ and reversed the cognitive deficits. Most importantly from a therapeutic perspective, blocking neutrophil adhesion during the early stages of the disease provided a long-term beneficial effect on cognition in older mice (19).

The presence of neutrophils in the brains of $\mathrm{AD}$ subjects was previously suggested by the presence of cells expressing cathepsin $\mathrm{G}$, a protease produced specifically by neutrophils, within the 
$\mathrm{AD}$ brain parenchyma and inside cerebral blood vessels (23). Additionally, the presence of CAP37, an inflammatory mediator expressed in neutrophils, was reported in the blood vessels and hippocampal vasculature of $\mathrm{AD}$ patients $(24,25)$. However, the first definitive evidence for the presence of neutrophils in the $\mathrm{AD}$ brain was our confirmation that myeloperoxidase (MPO) ${ }^{+}$ cells are localized in areas with $A \beta$ deposits (19). These cells were typically found around the periphery of $A \beta$ plaques at a distance of $<50 \mu \mathrm{m}$, and their non-random distribution suggested that $A \beta$ influences the microenvironmental positioning of neutrophils inside the $\mathrm{AD}$ brain.

Several reports indicate that $\mathrm{T}$ cells also accumulate in the $\mathrm{AD}$ brain (26-29). For example, a greater number of activated $\mathrm{CD}^{+}$and $\mathrm{CD} 8^{+} \mathrm{T}$ cells was observed in the blood, adhering to the vascular endothelium or migrating into the parenchyma of $\mathrm{AD}$ patients, compared to healthy controls or patients with other types of dementia $(4,26-29)$. Notably, the majority of $\mathrm{T}$ cells in $\mathrm{AD}$ brain tissue are located in the hippocampus and other limbic structures, which are among those regions most affected in $\mathrm{AD}$ (28). In support of these data, enhanced activated $\mathrm{CD} 4^{+}$and $\mathrm{CD} 8^{+}$ were recently identified in the cerebrospinal fluid (CSF) of individuals with mild cognitive impairment (MCI) and patients with mild $\mathrm{AD}$, with the proportion of activated $\mathrm{CD} 8^{+} \mathrm{T}$ cells showing the greatest increase (30). In agreement with human data, mouse studies have shown that $\mathrm{T}$ cells infiltrate the brains of APP/PS1 mice, and a proportion of these cells secrete interferon (IFN)- $\gamma$ or interleukin (IL)-17 (31). This suggests that the inflammatory response stimulated by $\mathrm{T}$ cells that have migrated into the $\mathrm{AD}$ brain may activate microglia and astrocytes and may recruit other inflammatory cells that are potentially harmful to the CNS, thus exacerbating the pathogenesis of $\mathrm{AD}$.

Taken together, the studies discussed in this section suggest that a role for circulating leukocytes in $\mathrm{AD}$ is becoming clearer, but further studies are needed to determine the impact of specific immune cell populations on the cognitive deficit and neuropathological changes in AD. In this context, future TPLSM studies may provide key data to increase our understanding of the mechanisms controlling leukocyte trafficking in the $\mathrm{AD}$ brain, as well as interactions between migrating leukocytes and CNSresident cells.

\section{TPLSM - THE GENERAL CONTEXT}

The optical principles of two-photon microscopy are based on the absorption of two longer-wavelength lower-energy photons as a single quantum of energy by a fluorophore, thus promoting an electron to an excited state (32). TPLSM offers several advantages over traditional forms of microscopy for the investigation of living systems because it provides three-dimensional deep-tissue images and single-cell spatiotemporal information that other imaging techniques cannot achieve (33-35). TPLSM is particularly suitable for high-resolution imaging in intact thick tissues, such as whole organs, brain slices, embryos, and live animals (intravital imaging). Extensive tissue penetration is possible due to the reduced scattering of the infrared (IR) excitation light compared to one-photon confocal microscopy. The restriction of two-photon excitation solely to the focal plane provides most of the advantages over traditional confocal microscopy. TPLSM generates fluorescence only within the focal plane, thus substantially reducing photobleaching and photodamage outside the excitation volume (which represents only a small proportion of the overall sample), and thereby prolonging the viability of specimens especially during long-term imaging.

The spatiotemporal dynamics of leukocyte trafficking can be investigated in vivo using cutting-edge TPLSM technology (36, 37). This technique has changed our static view of the immune system and allowed the dissection of leukocyte migration behavior and cell-cell contacts, which are fundamental requirements for an effective immune response $(38,39)$. Several aspects of leukocyte migration that could not be predicted using in vitro systems have been identified, thanks to this advanced technology, including leukocyte intravascular crawling and the directional movement and polarization of emigrated leukocytes along an extravascular chemokine gradient $(40,41)$.

\section{TPLSM IN THE AD BRAIN}

Two-photon laser scanning microscopy has recently contributed to several developments in the field of neuroscience, facilitating studies of cell morphology and function in the living brain. TPLSM has become increasingly necessary to study structural and functional changes in the living brain because the imaging of neurons, glia, and vasculature provides new insights into the function of the CNS under physiological and pathophysiological conditions (42-45). Initial studies investigated the structural plasticity of dendritic spines and the axons of pyramidal neurons in the mouse cortex and how their changes could influence long-term information storage $(46,47)$. TPLSM also revealed the dynamic structure of microglial cells that constantly survey the brain parenchyma and switch their behavior to an activated state immediately after injury (48). In addition to such morphological studies, TPLSM has also been used for calcium imaging, thus improving the analysis of neuronal signaling and plasticity $(49,50)$.

In the context of $\mathrm{AD}$, intravital imaging in the brain has mainly been used to study amyloid plaque deposition, dendritic spine loss, and microglial aggregation around $A \beta$ plaques $(6,51-55)$. TPLSM has allowed the repeated visualization of the same amyloid plaques labeled with fluorescent dyes to evaluate their growth in transgenic mouse models of $\operatorname{AD}(51,55,56)$. A refined TPLSM method was used to define the kinetics of amyloid plaque growth in Tg2576 mice (57). The simultaneous imaging of amyloid plaques and neurons labeled with viral-expressed green fluorescent protein (GFP) in a transgenic mouse model of $\mathrm{AD}$ highlighted the detrimental effect of $A \beta$ on the neuronal circuitry (58).

Recent TPLSM studies have confirmed that dendritic spine loss after newborn amyloid deposits persists in the proximity of amyloid plaques in APP/PS1 mice (59). TPLSM imaging has also revealed that the depletion of $\mathrm{A} \beta$ in the brains of PDAPP-YFP transgenic mice treated with anti- $\mathrm{A} \beta$ antibodies promotes the rapid recovery of existing amyloid-associated neuritic dystrophy in vivo, indicating that axonal and dendritic damage is a partially reversible phenomenon (60).

The availability of transgenic mice expressing fluorescent cells, such as microglia, has provided a more intimate view of the 
interactions between amyloid plaques, neuronal structures, and cells in the brain parenchyma during the progression of $\mathrm{AD}$ (61) or astrocytes (62). The rapid appearance of new amyloid plaques induces progressive changes in neurites, manifesting as dendritic and axonal abnormalities, and the activation and recruitment of microglia to areas with amyloid plaques (63).

Two-photon laser scanning microscopy has also facilitated the characterization of impaired microglial functionality near amyloid deposits in $\mathrm{AD}$ mice, suggesting a correlation between plaque deposition and microglial behavior (64).

Two-photon laser scanning microscopy analysis of leukocyte trafficking in the inflamed CNS has focused almost exclusively on infections, stroke, and experimental autoimmune encephalomyelitis (EAE) (65-69). However, recent TPLSM studies have shown that circulating leukocytes migrate into the brain parenchyma of AD mice, revealing previously unknown mechanisms of $\mathrm{AD}$ pathogenesis and helping to identify new therapeutic strategies for $\operatorname{AD}(19,20)$. Our recent TPLSM experiments have demonstrated that circulating neutrophils arrest and perform intraluminal crawling preferentially inside blood vessels with $A \beta$ deposits, supporting a role for $A \beta$ in leukocyte migration into the brain (19). Furthermore, we have recently shown using TPLSM that neutrophils isolated from LFA-1-deficient mice cannot adhere to the brain vessels and therefore extravasate in the brain parenchyma, suggesting that LFA-1 integrin is a key mediator of neutrophil trafficking in $\mathrm{AD}$ and that targeting this adhesion molecule may have a therapeutic effect. Future studies are necessary to better understand the dynamics of immune cell trafficking in $\mathrm{AD}$ and TPLSM experiments that allow the imaging and analysis of leukocyte trafficking in the $\mathrm{AD}$ brain are discussed below.

\section{IMAGING THE CORTEX IN AD MICE}

In vivo imaging in the cortex of $\mathrm{AD}$ mice can be achieved using deeply anesthetized animals with the head fixed on a stereotaxic device to reduce movement artifacts. Mice in deep anesthesia cannot maintain their core body temperature at $35-38^{\circ} \mathrm{C}$. Heat lamps or a heated stage are therefore required to maintain the correct temperature because leukocyte motility is temperature dependent (70). An incision is made along the midline of the scalp to expose the skull, and any fascia overlying the skull is scraped away (71). Two different surgical preparations can be used for intravital TPLSM in the mouse cortex: the thinned skull preparation and open cranial window. Both these methods have advantages and drawbacks depending on the purpose of the investigation.

The thinned skull technique involves thinning the calvarium to approximately $20-30 \mu \mathrm{m}$ leaving an intact and almost transparent periosteal layer. A circular region of 1-2 $\mathrm{mm}$ in diameter is prepared with a high-speed micro drill and/or a stainless steel burr above the somatosensory and motor cortex. Heat and vibration artifacts are minimized during drilling by the frequent application of cold saline solution or artificial CSF. Heating is also avoided by interrupting the drilling every few seconds. Bone dust is removed using compressed air.

The mouse skull consists of two thin layers of compact bone, sandwiching a thick layer of spongy tissue. This spongy bone contains tiny cavities arranged in concentric circles and multiple canaliculi that carry blood vessels. After removing the external compact bone, the middle layer of spongy bone is carefully thinned to approximately $75 \%$ of its original thickness. Some bleeding from the blood vessels running through the canaliculi may occur during thinning but usually stops spontaneously (72). The clear visualization of the pial vasculature gives an indication of the skull thickness, then thinning is continued manually using a microsurgical blade. This process is repeated until the bone in the central region becomes flexible and maximum image clarity is achieved (Figures 1A,B). Once the surgical procedure is complete, the thinned skull preparation can be imaged immediately (73). When imaging is completed, the wound margins of the scalp are closed using a nylon suture.

Skull thinning creates a translucent viewing window allowing the visualization of cells up to a depth of 200-300 $\mu \mathrm{m}$ below the pial surface, including the meninges and neocortex but not the deeper brain structures (48). Imaging through the thinned cranial window is a minimally invasive method allowing long acquisition sessions because the thinned skull still protects the brain from external changes in temperature and pressure $(74,75)$. Despite minor bleeding from diploic vessels, the thinned skull technique leaves the majority of anastomoses between the diploic vessels and dural vessels intact. However, thinning the skull to a specific depth over a large zone is technically challenging due to the curvature of the skull, and the area should therefore be no more than $3 \mathrm{~mm}$ in diameter to avoid damage to the underlying tissue (72). Furthermore, in the case of multiple acquisition sessions over time, re-thinning the excess bone deposition after few days is necessary to prevent skull regrowth occluding the preparation (73). Among the two available techniques, the thinned cranial preparation is better for the analysis of larger structures, such as cerebral vasculature, amyloid plaques, and leukocytes $(19,51,52,76)$.

The study of AD mechanisms in mice may benefit from the creation of a small break with the tip of a needle in the lateral wall at the site of the thinned skull preparation to allow the delivery of fluorophores into the brain, leaving the thinned region intact. The hole is then filled with sterile bone wax, and the animals are allowed to recover on a heating pad before being returned to their cages. This approach can be used, e.g., to allow the diffusion of anti-A $\beta$ antibodies directly labeled with fluorescein or thioflavin $S$, a sensitive and specific fluorescent reporter for the dense-core subset of senile plaques. This molecule has been used to label A $\beta$ deposits in transgenic mouse models of amyloid deposition, and the growth rate of $A \beta$ plaques and cerebral $A \beta$ angiopathy have been extensively monitored in vivo (51, 55, 76-78).

The in vivo imaging of senile plaques in $\mathrm{AD}$ mice can also be achieved using Methoxy-X04, which can be administered intravenously or intraperitoneally (79). Methoxy-X04 is a relatively small, lipophilic molecule that can enter the brain rapidly and in sufficient amounts to allow the sensitive and specific detection of $\mathrm{A} \beta$ deposits (79). Senile plaques and cerebrovascular $\mathrm{A} \beta$ angiopathy in $\mathrm{AD}$-like mice are visible $30 \mathrm{~min}$ after intravenous injection or $24 \mathrm{~h}$ after intraperitoneal injection (79). The intravenous or intraperitoneal administration of Methoxy-X04 is a more physiological approach to label $\mathrm{A} \beta$ in vivo compared to the creation of a small break in the skull near the thinning region to allow delivery of fluorophores into the brain. 

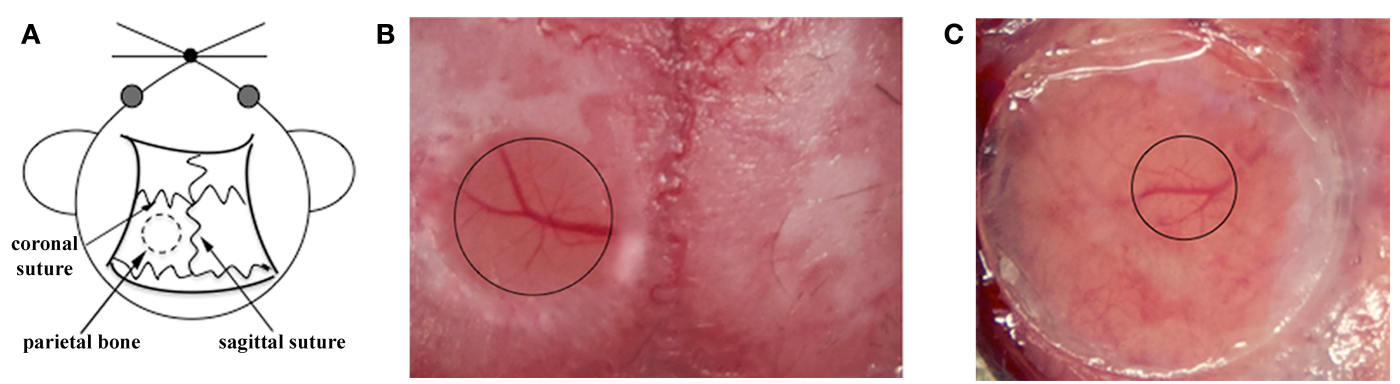

FIGURE 1 | Surgical procedures for TPLSM imaging of the brain cortex: thinned skull preparation and open cranial window. (A) For both procedures, a circular area of parietal bone is shaved with an electric razor and the scalp is sterilized with alcohol. The head of the anesthetized mouse is then fixed on a stereotaxic device to reduce movement artifacts during imaging, and the skull overlying the cortical region is exposed. (B) For the thinned skull preparation, a small region on the parietal zone of the skull is thinned using a high-speed micro drill and a stainless steel burr until the cortical vasculature can be seen clearly through the thinned skull. The thinned area is enclosed by the black circle. (C) For the open cranial window technique, a small island of cranial bone on the parietal region (black circle) is drilled, removed with a pair of sharp forceps, and covered with a circular glass coverslip.

The second major approach for TSPLM imaging is the open cranial window. The most challenging aspect of this technique is the surgical skill required for a successful preparation. In this procedure, a circular groove is drilled on the parietal region of the skull, and the island of cranial bone is carefully removed with a pair of sharp forceps. These should be held parallel to the skull surface as far as possible to avoid mechanical injury, because the dura can be attached to the overlying bone. Immediately after removing the region of the skull, slight bleeding above the dura may occur from small blood vessels attached to the removed skull fragment, but this should stop spontaneously within few seconds. The exposed brain tissue is preserved using a drop of $0.9 \% \mathrm{NaCl}$. A circular glass coverslip ( $5 \mathrm{~mm}$ in diameter) is placed over the incision site to cover the window (Figure 1C). After cementing the coverslip in place, mice are given a bolus of warm saline for rehydration and are allowed to recover from anesthesia for 7-14 days before imaging. The open cranial window technique also makes it unnecessary to introduce a needle-hole in the skull to deliver stains and labeling compounds. A recently described simple device provides easy physical access to the brain and allows long-term imaging (80). After the initial open cranial window surgery, a glass coverslip is applied as described above, but this has a pre-drilled access hole sealed with biocompatible silicone. This device allows many different types of manipulation to be carried out for weeks or months, e.g., drug, dye, and virus delivery, sample extraction, or electrophysiological recording and stimulation, while protecting the brain from infection (80).

The open cranial window provides improved optical access to the cortical layers, allowing repeated high-resolution imaging, and is preferable for the imaging of small structures, such as dendritic spines and filopodia $(73,81)$. However, removing the skull may induce a neuroinflammatory reaction and unavoidable meningeal vascular injury, involving the activation of microglia and astrocytes in the intact brain $(72,73,81,82)$. In this case, only cells lying deeper than $80 \mu \mathrm{m}$ below the pial surface should be considered for image analysis to eliminate possible artifacts caused by the surgical preparation.

To conduct multiple imaging sessions, some authors place a small metal bar containing a hole for a screw next to the coverslip to allow for repositioning of the mouse during subsequent imaging sessions. This approach has been used in Tg2576 mice for the long-term in vivo imaging to monitor individual amyloid plaques stained with Methoxy-X04 over a period of 6 weeks (55). Other studies using the same technique have followed individual $\mathrm{A} \beta$ plaques in Tg2576 mice for 5 months, confirming the biophysical model of $\mathrm{A} \beta$ plaque growth in vivo, which had been extrapolated from in vitro experiments.

Blood vessels can be labeled by the intravenous injection of fluorescent high-molecular-weight dextran $(>2000 \mathrm{kDa})$ or semiconductor nanocrystals (quantum dots), which suffer less interstitial leakage than dextrans (83) (Figure 2). Leukocytes can be labeled exvivo with vital dyes, such as 7-amino-4-chloromethylcoumarin (CMAC, blue), 5- (and 6-) carboxyfluorescein-diacetate succinimidyl-ester (CFSE, green), 5- (and 6-) (((4-chloromethyl) benzoyl)amino)tetramethylrhodamine (CMTMR, orange), $\mathrm{C}_{42} \mathrm{H}_{40} \mathrm{ClN}_{3} \mathrm{O}_{4}$ (CMTPX, red), and $\mathrm{C}_{33} \mathrm{H}_{24} \mathrm{~N}_{2} \mathrm{O}_{9}$ (SNARF-1, far red). The cells can then be adoptively transferred to syngeneic recipients allowing tracking for short time periods, usually a few days (Figure 2). Alternatively, the use of donor mice expressing fluorescent proteins ubiquitously is an option for the in vivo analysis of highly proliferative cell populations (84). Similarly, mice expressing fluorescent cell subsets allow the study of specific cell populations, such as neutrophils, lymphocytes, microglia, or macrophages $(61,85-87)$. Nowadays, mice engineered for the lineage-specific expression of GFP derivatives, such as eCFP and eYFP, or dsRed derivatives, such as tdTomato and mCherry, are widely used because they are less susceptible to phototoxicity and there is no need for cell isolation and labeling. However, most of these mice have been generated using GFP derivatives, limiting the ability to analyze several reporter genes in the same mouse simultaneously. Hence, new IR fluorescent dyes have been developed, which also facilitate deeper tissue imaging (88). The IR excitation wavelength requires the application of an optical parametric oscillator (OPO) to TPLSM. This allows live imaging for an extended duration because it reduces the photobleaching of fluorophores and phototoxicity-induced tissue damage.

The simultaneous expression of fluorescent proteins or staining using fluorescent dyes with different excitation spectra in the same 

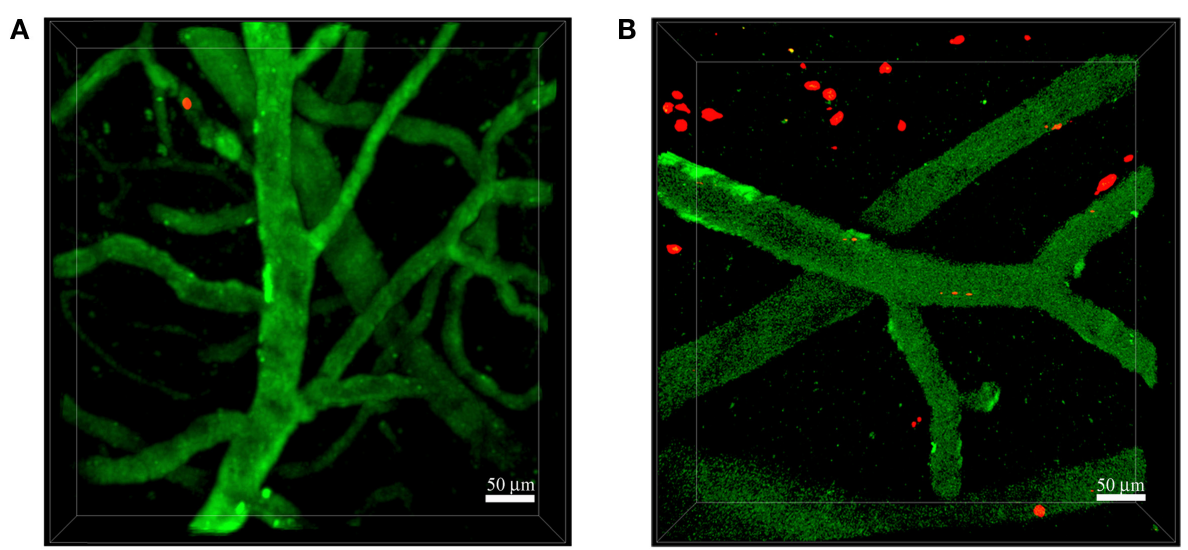

FIGURE 2 | Neutrophils invade the brain of 5xFAD mice. Representative TPLSM images of wild-type control mice (A) and 5xFAD mice (B) showing blood cortical vessels labeled in green using 525-nm non-targeted Qdots injected before image acquisition and neutrophils labeled in red with the fluorescent cell tracker CMTPX. The skull was exposed above the somatosensory cortex using the thinned skull preparation. We performed acquisition inside the brain parenchyma at a depth of approximately 150-250 $\mu \mathrm{m}$. Images were acquired 16-48 h after cell injection. (A) Neutrophils did not interact with the endothelium of blood vessels in wild-type control mice. (B) Numerous neutrophils migrated into the brain parenchyma of $5 x F A D$ mice. Scale bars $=50 \mu \mathrm{m}$.

mouse allows the in vivo visualization of interactions between specific cell populations and $A \beta$ deposits, thus enabling the study of $\mathrm{AD}$-specific cellular dynamics in the brain. This approach was recently used by our group to demonstrate that neutrophils migrate inside the parenchyma of $5 \mathrm{xFAD}-\mathrm{YFPH}$ mice in areas with $\mathrm{A} \beta$ plaques labeled with Methoxy-X04, probably driven by chemotactic factors, potentially including A $\beta$ peptides (19) (Figure 3). Our TPLSM studies also showed that the extravasation of neutrophils inside the brain of $5 \mathrm{xFAD}-\mathrm{YFPH}$ mice occurs in areas depleted for eYFP fluorescent neurons (Figure 3). We speculate that eYFP fluorescent neurons are turned off in the zones with neutrophil infiltration, probably due to the cytotoxic effects of proinflammatory mediators released by neutrophils, such as ROS, enzymes, neutrophil extracellular traps (NETs), and cytokines. Alternatively, this may reflect an indirect negative effect caused by the activation of microglia. Moreover, we have shown that neutrophils display arrest and intraluminal crawling preferentially inside blood vessels with labeled $A \beta$ deposits and that some cells undergo diapedesis adjacent to vascular $\mathrm{A} \beta$ deposits (19). TPLSM experiments performed by others have shown that neutrophils are attracted inside the brain parenchyma by chronic $A \beta$ deposition - initially the cells move randomly outside the vessels and then they are suddenly and massively recruited to specific A $\beta$ plaques in the brain parenchyma (20).

Two-photon laser scanning microscopy can be used to study CNS-resident cells (such as neuronal subsets, astrocytes, microglia, and perivascular macrophages) in relation to each other or in relation to infiltrating leukocytes. TPLSM studies have shown that local resident microglia react to $\mathrm{A} \beta$ plaque formation by extending processes and subsequently migrating toward plaques in APP/PS1 mice crossed with mice containing fluorescent microglia (53). Furthermore, astrocytes in the mouse neocortex can be visualized in vivo by intravenous injection of the non-toxic molecule sulforhodamine B or by using transgenic mice, in which astrocytes express enhanced GFP under the control of the mouse glial fibrillary acidic protein (GFAP) promoter $(89,90)$. TPLSM in vivo imaging of astrocyte $\mathrm{Ca}^{2+}$ signaling revealed abnormalities in astrocyte activity that may contribute to vascular instability and thereby to neuronal cell death in several transgenic mouse models of AD (91). However, whether infiltrating leukocytes interact with resident neural cells is completely unknown in AD and future studies focusing on such interactions will be necessary to discover and understand these new disease mechanisms.

\section{IMAGE ACQUISITION AND ANALYSIS}

During image acquisition, mice are deeply anesthetized with isoflurane and the rate of respiration is controlled. The laser intensity and photomultiplier tube (PMT) gain need to be set carefully to minimize photodamage. The imaging volume and sampling frequency must be chosen to ensure successful image analysis with fine resolution in time and space. Stacks of optical sections are serially re-acquired at defined time intervals, and cell centroids need to be determined in order to track cell motion. The tracks locate the cells at each time point. They consist of serial sets of $x y z$ coordinates of single-cell centroids. The tracks are then exported as numerical data and are used to calculate specific parameters for the analysis of cell migration (36). Specialized software packages are used for automated 3D cell tracking and the analysis of migration paths for each cell. The automatic tracking and analysis of individual cells is more reliable when a small proportion of cells is labeled, because this reduces the likelihood of overlapping pixels representing different cells and allows the distinction of individual cells based on their centers of mass.

Several parameters can be used to analyze the migration behavior of cells during TPLSM experiments. Cell velocity can be represented as either instantaneous velocity or track velocity. Instantaneous velocity is a basic parameter derived from the displacement of the cell divided by the elapsed time (70). The track velocity is calculated from the median or mean instantaneous 
A

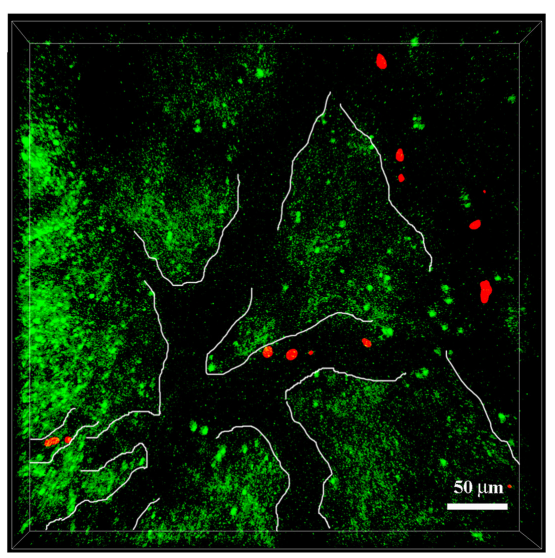

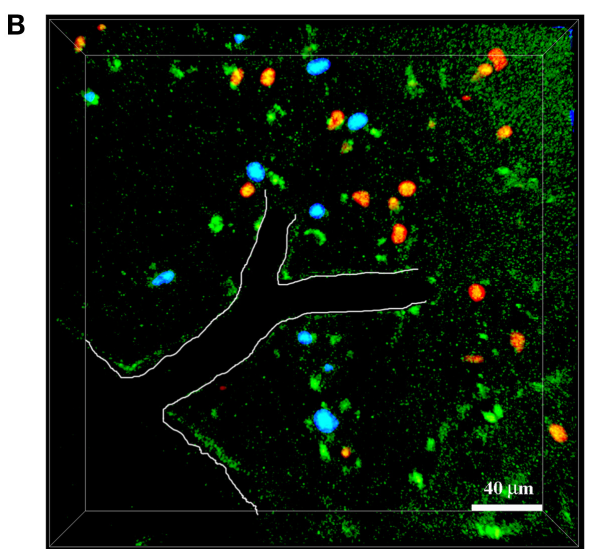

FIGURE 3 | Neutrophils infiltrate the brain of 5xFAD-YFPH mice in A $\beta$-rich areas. Representative TPLSM images of cortical regions in 5xFAD-YFPH mice showing YFP neurons in green, neutrophils in red stained with cell tracker CMTPX, and A $\beta$ plaques in blue labeled by the intravenous injection of Methoxy-X04. The vessel edges are traced artificially with a white line to show the vessel bed. (A) Neutrophils interact with the vascular endothelium. Scale bar $=50 \mu \mathrm{m}$. (B) Neutrophils infiltrate into the brain parenchyma, characterized by abundant A $\beta$ plaques and weak neuronal fluorescence. Scale bar $=40 \mu \mathrm{m}$.

velocity computed from all time intervals throughout a track, typically 6-14 time points at intervals of 20-50 s (92). Because leukocytes migrate along stromal cell networks, they do not travel along a linear path but instead make frequent turns in a "random walk." Therefore, higher sampling frequencies are necessary to provide an accurate readout of instantaneous velocities and to avoid the underestimation of real velocity when cells are persistent for only a finite period of time (93). It is easy to find differences in the motility parameters of leukocytes reported in previous studies, and such differences are likely to reflect the frame rate of data acquisition. If there is a long gap between each frame, then the true velocity of the cell will be underestimated. Therefore, the "fast sampling" theory (the use of sampling intervals shorter than the persistence time of the cell) should be taken into account in order to correctly compare data obtained in different laboratories (93).

A useful parameter that is derived from velocity is the arrest coefficient, representing the fraction of time during tracking in which a cell does not move (threshold $<2 \mu \mathrm{m} / \mathrm{min}$ ). It is calculated as the ratio of the time a cell is immotile over the whole observation time. The arrest coefficient is calculated from cell tracks and reported as the percentage of cells in the entire population (70). The arrest coefficient is high when leukocytes are in stable contact with other cells or when they swarm in a chemoattractant microenvironment (94).

The cell locomotion parameter allows some speculation on the nature of cell movement, e.g., cells may show directed migration along a gradient of a soluble or surface-immobilized chemoattractant. The displacement of a cell moving with a constant velocity is the shortest distance between the positions at two time points (95). An accurate parameter to analyze migration patterns is the mean displacement (MD) plot (70), in which the average displacement of a population of cells over specific time intervals is plotted against the square root of time. The slope of the resulting curve can be used to determine the motility coefficient of a cell population and measures the volume that a cell scans per unit time. Cells usually exhibit directed movement for a few minutes, which means that during short time intervals, they tend to move in one preferred direction (36).

The chemotactic index, also called the meandering index or straightness index, is another parameter representing the confinement of cell tracks and is calculated based on the ratio of displacement from origin by track length. The meandering can vary between 0 (the cell returns to its exact starting position) and 1 (a perfectly straight cell track). Cells exhibiting frequent angle changes will produce tracks with low meandering indices, whereas a meandering index of $0.7-1$ generally shows that cell migration has a strong directional bias (70).

During time-lapse acquisition, a moving cell is observed by taking snapshots at fixed time intervals; hence, the movement of each cell might be considered as a sequence of vectors. Trajectory vectors represent the direction of displacement of individual cells, so the calculation of angles between the direction of migration and various other directions in space is possible, e.g., the orientation of a blood vessel. The vector-vessel angles of individual tracks are useful for the description of tangential movement along an axis and are determined by mirroring the trajectory vectors onto the nearest vessel (reference vector), resulting in angles of $0-90^{\circ}$ for each cell track (96).

It is important to realize that cell populations analyzed using the parameters described above may consist of distinct subpopulations that have different biological roles and migration behaviors. These subpopulations may be revealed by plotting the distribution of the parameter of interest among all cells or by studying correlations between multiple motility parameters. For example, neutrophils invading the brain parenchyma in AD-like mice show two distinct behaviors: the first involves a strong directional bias characterized by a high meandering index, motility coefficient, and mean track velocity, whereas the second is characterized by low motility and undirected movement resembling swarming behavior (19). The undirected motility behavior with numerous cells displaying full arrest indicates the presence of activating stop signals for neutrophils in the brains of 
mice with AD-like pathology, whereas the directed movement of neutrophils strongly suggests the presence of chemotactic factors (19).

Other parameters calculated from leukocyte intratissue migration may reveal whether a chemoattractant released by injured tissue, resident cells, or recruited blood-derived leukocytes direct their movement. For example, after entering peripheral tissues, neutrophils assume an amoeboid motility profile characterized by coordinated migration along a chemokine gradient and cluster formation. The dramatic changes in neutrophil morphology can be analyzed by shape index (length/width), revealing the significant elongation of cell bodies, which is the characteristic of adhesion-independent movement associated with a low degree of cytoskeletal organization and a lack of discrete focal contacts (97).

The directional component of migration can be determined by measuring the turning angles of neutrophil tracks, which is achieved by calculating the angle change between vectors constructed from each time point. The peak angle distribution in absolute values varies in the range $0-180^{\circ}$, and changes of $10-30$ units indicate highly directed migration following a chemotactic gradient (97). In order to identify specific chemotactic molecules that direct leukocyte movement, instantaneous radial velocities can be calculated for the comparative chemotaxis analysis of wild-type and gene-deficient neutrophil populations (98). The radial velocity-time plot with fitted regression lines can provide insight into the recruitment dynamics of cell populations, and a reduction in radial velocity may be a consequence of impaired chemotaxis (98). Although no data are yet available in AD models, integrin-dependent intravascular neutrophil migration in sterile liver injuries requires CXCR2 ligands on liver sinusoids and formyl peptides in the injury zone (99). Furthermore, in sterile skin injuries, leukotriene B4 acts as an intercellular signal between neutrophils that allows rapid integrin-independent neutrophil recruitment throughout the tissue (98). Future studies are needed to characterize the dynamics of neutrophils as well as other leukocyte populations in the brains of $\mathrm{AD}$ models.

\section{FUTURE DIRECTIONS}

Two-photon laser scanning microscopy has made it possible to study leukocyte recruitment in the living brain, improving our understanding of immune cell functions in CNS diseases, such as $\mathrm{AD}$. Advanced imaging techniques will also allow us to define the role of leukocyte subpopulations at different stages of disease, as well as the relationships among migrating leukocytes, amyloid deposition, and tau pathology. Moreover, it is conceivable that TPLSM in the AD brain will help us to visualize and analyze the interplay between migrating leukocytes and resident cells, such as microglia, neurons, and astrocytes. TPLSM could also be used to unravel the role of toxic molecules, such as ROS and NO intermediates that may be produced by invading innate immunity cells during AD. For example, dihydroethidium and hydroethidine are cell-permeable fluorescent dyes suitable for the evaluation of ROS synthesis in vitro, but they have also been used successfully to visualize anion superoxide production in vivo $(100,101)$. The development of new fluorescent probes and the deep-tissue imaging capability of TPLSM may allow the in vivo visualization of ROS production by neural cells and infiltrating leukocytes.

One of the limitations of current imaging studies is the lack of access to deeper areas of the intact brain, such as the hippocampus, which is strongly affected during AD. Nevertheless, recently developed micro-optics and micro-mechanical components have improved deep-tissue imaging $(102,103)$. Indeed, the use of micro-prisms and gradient index (GRIN) lenses allows the longterm imaging of deep-layer cortical tissue in the mouse brain, although such devices must be inserted into the parenchyma, potentially altering neural cell functions and inducing inflammatory responses $(102,104)$. GRIN lenses feature plane optical surfaces and are focused by continuously changing the refractive index within the lens material by eliminating aberrations typically found in traditional spherical lenses. An alternative deep-tissue imaging method is stimulated emission depletion (STED) laser microscopy, which allows the exploration of deep areas, such as the hippocampus in living brains (105). This recent imaging technique improves spatial resolution by quenching the fluorescence everywhere except the central region, thus substantially reducing the size of the original fluorescence spot. Therefore, the higher peak signal-to-background ratio reveals more detailed structures, improving the quality of the images. In the near future, STED laser microscopy may expand to include the analysis of leukocyte recruitment in key brain areas during $\mathrm{AD}$, leading to the identification of new disease mechanisms. Moreover, STED technology brings in vivo super-resolution microscopy to small structures involved in the morphofunctional interactions between astrocytes and neurons, suggesting future exciting opportunities for the study of leukocyte interactions with neural cells (106).

Overall, the augmentation of TPLSM using optical systems, such as GRIN lenses or STED laser microscopy, and advances in fluorescent dye and protein engineering to produce brighter and more photostable fluorophores will play an important role in the study of molecular mechanisms controlling leukocytedependent brain injury and will help to identify new therapeutic approaches for $\mathrm{AD}$.

\section{ETHICS STATEMENT}

All mouse experiments were carried out in accordance with guidelines prescribed by the Ethics Committee for the usage of laboratory animals for research purposes at the University of Verona and by the Italian Ministry of Health.

\section{AUTHOR CONTRIBUTIONS}

All authors contributed equally.

\section{FUNDING}

This work was supported in part by the European Research Council grants 261079 NEUROTRAFFICKING and 693606 IMPEDE; National Multiple Sclerosis Society (NMSS), New York, NY, USA; Fondazione Cariverona; Italian Ministry of Health grant GR2009; Italian Ministry of Education and Research (MIUR); and Fondazione Italiana Sclerosi Multipla (FISM) (to GC). 


\section{REFERENCES}

1. Querfurth HW, LaFerla FM. Alzheimer's disease. N Engl J Med (2010) 362:329-44. doi:10.1056/NEJMra0909142

2. Wisniewski T, Goñi F. Immunotherapeutic approaches for Alzheimer's disease. Neuron (2015) 85:1162-76. doi:10.1016/j.neuron.2014.12.064

3. Heppner FL, Ransohoff RM, Becher B. Immune attack: the role of inflammation in Alzheimer disease. Nat Rev Neurosci (2015) 16:358-72. doi:10.1038/nrn3880.3

4. Rossi B, Angiari S, Zenaro E, Budui SL, Constantin G. Vascular inflammation in central nervous system diseases: adhesion receptors controlling leukocyte-endothelial interactions. J Leukoc Biol (2011) 89:539-56. doi:10.1189/jlb.0710432

5. Ley K, Laudanna C, Cybulsky MI, Nourshargh S. Getting to the site of inflammation: the leukocyte adhesion cascade updated. Nat Rev Immunol (2007) 7:678-89. doi:10.1038/nri2156

6. El Khoury J, Toft M, Hickman SE, Means TK, Terada K, Geula C, et al. Ccr2 deficiency impairs microglial accumulation and accelerates progression of Alzheimer-like disease. Nat Med (2007) 13:432-8. doi:10.1038/nm1555

7. Simard AR, Soulet D, Gowing G, Julien JP, Rivest S. Bone marrow-derived microglia play a critical role in restricting senile plaque formation in Alzheimer's disease. Neuron (2006) 49:489-502. doi:10.1016/j. neuron.2006.01.022

8. Town T, Laouar Y, Pittenger C, Mori T, Szekely CA, Tan J, et al. Blocking TGFbeta-Smad $2 / 3$ innate immune signaling mitigates Alzheimer-like pathology. Nat Med (2008) 14:681-7. doi:10.1038/nm1781

9. Lebson L, Nash K, Kamath S, Herber D, Carty N, Lee DC, et al. Trafficking CD11b-positive blood cells deliver therapeutic genes to the brain of amyloid-depositing transgenic mice. J Neurosci (2010) 30:9651-8. doi:10.1523/ JNEUROSCI.0329-10.2010

10. Mildner A, Schmidt H, Nitsche M, Merkler D, Hanisch UK, Mack M, et al. Microglia in the adult brain arise from Ly-6ChiCCR2+ monocytes only under defined host conditions. Nat Neurosci (2007) 10:1544-53. doi:10.1038/ nn2015

11. Fiala M, Zhang L, Gan X, Sherry B, Taub D, Graves MC, et al. Amyloidbeta induces chemokine secretion and monocyte migration across a human blood-brain barrier model. Mol Med (1998) 4:480-9.

12. Giri R, Shen Y, Stins M, Du Yan S, Schmidt AM, Stern D, et al. Beta-amyloidinduced migration of monocytes across human brain endothelial cells involves RAGE and PECAM-1. Am J Physiol Cell Physiol (2000) 279:C1772-81.

13. Simard AR, Rivest $\mathrm{S}$. Bone marrow stem cells have the ability to populate the entire central nervous system into fully differentiated parenchymal microglia. FASEB J (2004) 18:998-1000. doi:10.1096/fi.04-1517fje

14. Jay TR, Miller CM, Cheng PJ, Graham LC, Bemiller S, Broihier ML, et al. TREM2 deficiency eliminates TREM2+ inflammatory macrophages and ameliorates pathology in Alzheimer's disease mouse models. J Exp Med (2015) 212:287-95. doi:10.1084/jem.20142322

15. Michaud JP, Bellavance MA, Préfontaine P, Rivest S. Real-time in vivo imaging reveals the ability of monocytes to clear vascular amyloid beta. Cell Rep (2013) 5:646-53. doi:10.1016/j.celrep.2013.10.010

16. Zarbock A, Ley K. Mechanisms and consequences of neutrophil interaction with the endothelium. Am J Pathol (2008) 172:1-7. doi:10.2353/ ajpath.2008.070502

17. DiStasi MR, Ley K. Opening the flood-gates: how neutrophil-endothelial interactions regulate permeability. Trends Immunol (2009) 30:547-56. doi:10.1016/j.it.2009.07.012

18. Fabene PF, Navarro Mora G, Martinello M, Rossi B, Merigo F, Ottoboni L, et al. A role for leukocyte-endothelial adhesion mechanisms in epilepsy. Nat Med (2008) 14:1377-83. doi:10.1038/nm.1878

19. Zenaro E, Pietronigro E, Della Bianca V, Piacentino G, Marongiu L, Budui S. Neutrophils promote Alzheimer's disease-like pathology and cognitive decline via LFA-1 integrin. Nat Med (2015) 21:880-6. doi:10.1038/nm.3913

20. Baik SH, Cha MY, Hyun YM, Cho H, Hamza B, Kim DK, et al. Migration of neutrophils targeting amyloid plaques in Alzheimer's disease mouse model. NeurobiolAging(2014)35:1286-92.doi:10.1016/j.neurobiolaging.2014.01.003

21. Joslin G, Griffin GL, August AM, Adams S, Fallon RJ, Senior RM, et al. The serpin-enzyme complex (SEC) receptor mediates the neutrophil chemotactic effect of alpha-1 antitrypsin-elastase complexes and amyloid-beta peptide. J Clin Invest (1992) 90:1150-4. doi:10.1172/JCI115934
22. Tiffany HL, Lavigne MC, Cui YH, Wang JM, Leto TL, Gao JL, et al. Amyloidbeta induces chemotaxis and oxidant stress by acting at formylpeptide receptor 2, a G protein-coupled receptor expressed in phagocytes and brain. J Biol Chem (2001) 276:23645-52. doi:10.1074/jbc.M101031200

23. Savage MJ, Iqbal M, Loh T, Trusko SP, Scott R, Siman R. Cathepsin G: localization in human cerebral cortex and generation of amyloidogenic fragments from the beta-amyloid precursor protein. Neuroscience (1994) 60:607-19. doi:10.1016/0306-4522(94)90490-1

24. Grammas P. A damaged microcirculation contributes to neuronal cell death in Alzheimer's disease. Neurobiol Aging (2000) 21:199-205. doi:10.1016/ S0197-4580(00)00102-0

25. Brock AJ, Kasus-Jacobi A, Lerner M, Logan S, Adesina AM, Anne Pereira $\mathrm{H}$. The antimicrobial protein, CAP37, is upregulated in pyramidal neurons during Alzheimer's disease. Histochem Cell Biol (2015) 144:293-308. doi:10.1007/s00418-015-1347-x

26. Itagaki S, McGeer PL, Akiyama H. Presence of T-cytotoxic suppressor and leucocyte common antigen positive cells in Alzheimer's disease brain tissue. Neurosci Lett (1988) 91:259-64. doi:10.1016/0304-3940(88)90690-8

27. Rogers J, Luber-Narod J, Styren SD, Civin WH. Expression of immune system-associated antigens by cells of the human central nervous system: relationship to the pathology of Alzheimer's disease. Neurobiol Aging (1988) 9:339-49. doi:10.1016/S0197-4580(88)80079-4

28. Togo T, Akiyama H, Iseki E, Kondo H, Ikeda K, Kato M, et al. Occurrence of T cells in the brain of Alzheimer's disease and other neurological diseases. J Neuroimmunol (2002) 124:83-92. doi:10.1016/S0165-5728(01)00496-9

29. Town T, Tan J, Flavell RA, Mullan M. T-cells in Alzheimer's disease. Neuromol Med (2005) 7:255-64. doi:10.1385/NMM:7:3:255

30. Lueg G, Gross CC, Lohmann H, Johnen A, Kemmling A, Deppe M, et al. Clinical relevance of specific T-cell activation in the blood and cerebrospinal fluid of patients with mild Alzheimer's disease. Neurobiol Aging (2015) 36:81-9. doi:10.1016/j.neurobiolaging.2014.08.008

31. Browne TC, McQuillan K, McManus RM, O’Reilly JA, Mills KH, Lynch MA. IFN- $\gamma$ production by amyloid $\beta$-specific Th1 cells promotes microglial activation and increases plaque burden in a mouse model of Alzheimer's disease. J Immunol (2013) 190:2241-51. doi:10.4049/jimmunol.1200947

32. Denk W, Strickler JH, Webb WW. Two-photon laser scanning fluorescence microscopy. Science (1990) 248:73-6. doi:10.1126/science.2321027

33. Helmchen F. Chapter 2. Two-photon functional imaging of neuronal activity. In: Frostig RD, editor. In Vivo Optical Imaging of Brain Function. 2nd ed. Boca Raton, FL: CRC Press; Taylor \& Francis (2009). p. 37-58.

34. Helmchen F, Denk W. Deep tissue two-photon microscopy. Nat Methods (2005) 2:932-40. doi:10.1038/nmeth818

35. Svoboda K, Yasuda R. Principles of two-photon excitation microscopy and its applications to neuroscience. Neuron (2006) 50:823-39. doi:10.1016/j. neuron.2006.05.019

36. Sumen C, Mempel TR, Mazo IB, von Andrian UH. Intravital microscopy: visualizing immunity in context. Immunity (2004) 21:315-29. doi:10.1016/j. immuni.2004.08.006

37. Henrickson SE, von Andrian UH. Single-cell dynamics of T-cell priming. Curr Opin Immunol (2007) 19:249-58. doi:10.1016/j.coi.2007.04.013

38. Germain RN, Robey EA, Cahalan MD. A decade of imaging cellular motility and interaction dynamics in the immune system. Science (2012) 336:1676-81. doi:10.1126/science. 1221063

39. Balagopalan L, Sherman E, Barr VA, Samelson LE. Imaging techniques for assaying lymphocyte activation in action. Nat Rev Immunol (2011) 11:21-33. doi:10.1038/nri2903

40. Kolaczkowska E, Kubes P. Neutrophil recruitment and function in health and inflammation. Nat Rev Immunol (2013) 13:159-75. doi:10.1038/nri3399

41. Phillipson M, Kubes P. The neutrophil in vascular inflammation. Nat Med (2011) 17:1381-90. doi:10.1038/nm.2514

42. Grutzendler J, Gan WB. Two-photon imaging of synaptic plasticity and pathology in the living mouse brain. NeuroRx (2006) 3:489-96. doi:10.1016/j. nurx.2006.07.005

43. Grutzendler J, Helmin K, Tsai J, Gan WB. Various dendritic abnormalities are associated with fibrillar amyloid deposits in Alzheimer's disease. Ann $\mathrm{N}$ Y Acad Sci (2007) 1097:30-9. doi:10.1196/annals.1379.003

44. Bhatt DH, Zhang S, Gan WB. Dendritic spine dynamics. Annu Rev Physiol (2009) 71:261-82. doi:10.1146/annurev.physiol.010908.163140 
45. Nayak D, Zinselmeyer BH, Corps KN, McGavern DB. In vivo dynamics of innate immune sentinels in the CNS. Intravital (2012) 1:95-106. doi:10.4161/ intv. 22823

46. Trachtenberg JT, Chen BE, Knott GW, Feng G, Sanes JR, Welker E, et al. Long-term in vivo imaging of experience-dependent synaptic plasticity in adult cortex. Nature (2002) 420:788-94. doi:10.1038/nature01273

47. Chklovskii DB, Mel BW, Svoboda K. Cortical rewiring and information storage. Nature (2004) 431:782-8. doi:10.1038/nature03012

48. Nimmerjahn A, Kirchhoff F, Helmchen F. Resting microglial cells are highly dynamic surveillants of brain parenchyma in vivo. Science (2005) 308:1314-8. doi:10.1126/science.1110647

49. Grienberger C, Konnerth A. Imaging calcium in neurons. Neuron (2012) 73:862-85. doi:10.1016/j.neuron.2012.02.011

50. Stosiek C, Garaschuk O, Holthoff K, Konnerth A. In vivo two-photon calcium imaging of neuronal networks. Proc Natl Acad Sci U S A (2003) 100:7319-24. doi:10.1073/pnas.1232232100

51. Christie RH, Bacskai BJ, Zipfel WR, Williams RM, Kajdasz ST, Webb WW, et al. Growth arrest of individual senile plaques in a model of Alzheimer's disease observed by in vivo multiphoton microscopy. J Neurosci (2001) 21:858-64.

52. Tsai J, Grutzendler J, Duff K, Gan WB. Fibrillar amyloid deposition leads to local synaptic abnormalities and breakage of neuronal branches. Nat Neurosci (2004) 7:1181-3. doi:10.1038/nn1335

53. Bolmont T, Haiss F, Eicke D, Radde R, Mathis CA, Klunk WE, et al. Dynamics of the microglial/amyloid interaction indicate a role in plaque maintenance. J Neurosci (2008) 28:4283-92. doi:10.1523/JNEUROSCI.4814-07.2008

54. Bittner T, Fuhrmann M, Burgold S, Ochs SM, Hoffmann N, Mitteregger $\mathrm{G}$, et al. Multiple events lead to dendritic spine loss in triple transgenic Alzheimer's disease mice. PLoS One (2010) 5:e15477. doi:10.1371/journal. pone. 0015477

55. Burgold S, Bittner T, Dorostkar MM, Kieser D, Fuhrmann M, Mitteregger G, et al. In vivo multiphoton imaging reveals gradual growth of newborn amyloid plaques over weeks. Acta Neuropathol (2011) 121:327-35. doi:10.1007/ s00401-010-0787-6

56. Hefendehl JK, Wegenast-Braun BM, Liebig C, Eicke D, Milford D, Calhoun $\mathrm{ME}$, et al. Long-term in vivo imaging of beta-amyloid plaque appearance and growth in a mouse model of cerebral beta-amyloidosis. J Neurosci (2011) 31:624-9. doi:10.1523/JNEUROSCI.5147-10.2011

57. Burgold S, Filser S, Dorostkar MM, Schmidt B, Herms J. In vivo imaging reveals sigmoidal growth kinetic of $\beta$-amyloid plaques. Acta Neuropathol Commun (2014) 2:30. doi:10.1186/2051-5960-2-30

58. Spires TL, Meyer-Luehmann M, Stern EA. Dendritic spine abnormalities in amyloid precursor protein transgenic mice demonstrated by gene transfer and intravital multiphoton microscopy. JNeurosci (2005) 25:7278-87. doi:10.1523/JNEUROSCI.1879-05.2005

59. Bittner T, Burgold S, Dorostkar MM, Fuhrmann M, Wegenast-Braun BM, Schmidt B, et al. Amyloid plaque formation precedes dendritic spine loss. Acta Neuropathol (2012) 124:797-807. doi:10.1007/s00401-012-1047-8

60. Brendza RP, Bacskai BJ, Cirrito JR, Simmons KA, Skoch JM, Klunk WE, et al. Anti-Abeta antibody treatment promotes the rapid recovery of amyloid-associated neuritic dystrophy in PDAPP transgenic mice. J Clin Invest (2005) 115:428-33. doi:10.1172/JCI23269

61. Jung S, Aliberti J, Graemmel P, Sunshine MJ, Kreutzberg GW, Sher A, et al. Analysis of fractalkine receptor CX(3)CR1 function by targeted deletion and green fluorescent protein reporter gene insertion. Mol Cell Biol (2000) 20:4106-14. doi:10.1128/MCB.20.11.4106-4114.2000

62. Zuo Y, Lubischer JL, Kang H, Tian L, Mikesh M, Marks A, et al. Fluorescent proteins expressed in mouse transgenic lines mark subsets of glia, neurons, macrophages, and dendritic cells for vital examination. JNeurosci (2004) 24:10999-1009. doi:10.1523/JNEUROSCI.3934-04.2004

63. Meyer-Luehmann M, Spires-Jones TL, Prada C, Garcia-Alloza M, de Calignon A, Rozkalne A, et al. Rapid appearance and local toxicity of amyloid-beta plaques in a mouse model of Alzheimer's disease. Nature (2008) 451:720-4. doi:10.1038/nature06616

64. Krabbe G, Halle A, Matyash V, Rinnenthal JL, Eom GD, Bernhardt U, et al. Functional impairment of microglia coincides with beta-amyloid deposition in mice with Alzheimer-like pathology. PLoS One (2013) 8:e60921. doi:10.1371/journal.pone.0060921
65. Mrass $\mathrm{P}$, Weninger W. Immune cell migration as a means to control immune privilege: lessons from the CNS and tumors. Immunol Rev (2006) 213:195-212. doi:10.1111/j.1600-065X.2006.00433.x

66. Bartholomäus I, Kawakami N, Odoardi F, Schläger C, Miljkovic D, Ellwart JW, et al. Effector T cell interactions with meningeal vascular structures in nascent autoimmune CNS lesions. Nature (2009) 462:94-8. doi:10.1038/ nature 08478

67. Coombes JL, Robey EA. Dynamic imaging of host-pathogen interactions in vivo. Nat Rev Immunol (2010) 10:353-64. doi:10.1038/nri2746

68. Kawakami N, Flügel A. Knocking at the brain's door: intravital two-photon imaging of autoreactive $\mathrm{T}$ cell interactions with CNS structures. Semin Immunopathol (2010) 32:275-87. doi:10.1007/s00281-010-0216-X

69. McGavern DB, Kang SS. Illuminating viral infections in the nervous system. Nat Rev Immunol (2011) 11:318-29. doi:10.1038/nri2971

70. Zinselmeyer BH, Dempster J, Wokosin DL, Cannon JJ, Pless R, Parker I, et al. Chapter 16. Two-photon microscopy and multidimensional analysis of cell dynamics. Methods Enzymol (2009) 461:349-78. doi:10.1016/ S0076-6879(09)05416-0

71. Zenaro E, Rossi B, Angiari S, Constantin G. Use of imaging to study leukocyte trafficking in the central nervous system. Immunol Cell Biol (2013) 91:271-80. doi:10.1038/icb.2012.81

72. Xu HT, Pan F, Yang G, Gan WB. Choice of cranial window type for in vivo imaging affects dendritic spine turnover in the cortex. Nat Neurosci (2007) 10:549-51. doi:10.1038/nn1883

73. Yang G, Pan F, Parkhurst CN, Grutzendler J, Gan WB. Thinned-skull cranial window technique for long-term imaging of the cortex in live mice. Nat Protoc (2010) 5:201-8. doi:10.1038/nprot.2009.222

74. Kim JV, Jiang N, Tadokoro CE, Liu L, Ransohoff RM, Lafaille JJ, et al. Two-photon laser scanning microscopy imaging of intact spinal cord and cerebral cortex reveals requirement for CXCR6 and neuroinflammation in immune cell infiltration of cortical injury sites. J Immunol Methods (2010) 352:89-100. doi:10.1016/j.jim.2009.09.007

75. Davalos D, Lee JK, Smith WB, Brinkman B, Ellisman MH, Zheng B, et al. Stable in vivo imaging of densely populated glia, axons and blood vessels in the mouse spinal cord using two-photon microscopy. J Neurosci Methods (2008) 169:1-7. doi:10.1016/j.jneumeth.2007.11.011

76. Yoder EJ, Kleinfeld D. Cortical imaging through the intact mouse skull using two-photon excitation laser scanning microscopy. Microsc Res Tech (2002) 56:304-5. doi:10.1002/jemt.10002

77. D’Amore JD, Kajdasz ST, McLellan ME, Bacskai BJ, Stern EA, Hyman BT. In vivo multiphoton imaging of a transgenic mouse model of Alzheimer disease reveals marked thioflavine-S-associated alterations in neurite trajectories. J Neuropathol Exp Neurol (2003) 62:137-45. doi:10.1093/jnen/62.2.137

78. Dong J, Revilla-Sanchez R, Moss S, Haydon PG. Multiphoton in vivo imaging of amyloid in animal models of Alzheimer's disease. Neuropharmacology (2010) 59:268-75. doi:10.1016/j.neuropharm.2010.04.007

79. Klunk WE, Bacskai BJ, Mathis CA, Kajdasz ST, McLellan ME, Frosch MP, et al. Imaging Abeta plaques in living transgenic mice with multiphoton microscopy and methoxy-X04, a systemically administered congo red derivative. J Neuropathol Exp Neurol (2002) 61:797-805. doi:10.1093/jnen/61.9.797

80. Roome CJ, Kuhn B. Chronic cranial window with access port for repeated cellular manipulations, drug application, and electrophysiology. Front Cell Neurosci (2014) 8:379. doi:10.3389/fncel.2014.00379

81. Majewska AK, Newton JR, Sur M. Remodeling of synaptic structure in sensory cortical areas in vivo. JNeurosci (2006) 26:3021-9. doi:10.1523/ JNEUROSCI.4454-05.2006

82. Roth TL, Nayak D, Atanasijevic T, Koretsky AP, Latour LL, McGavern DB. Transcranial amelioration of inflammation and cell death after brain injury. Nature (2014) 505:223-8. doi:10.1038/nature12808

83. Stroh M, Zimmer JP, Duda DG, Levchenko TS, Cohen KS, Brown EB, et al. Quantum dots spectrally distinguish multiple species within the tumor milieu in vivo. Nat Med (2005) 11:678-82. doi:10.1038/nm1247

84. Luche H, Weber O, Nageswara Rao T, Blum C, Fehling HJ. Faithful activation of an extra-bright red fluorescent protein in "knock-in" Cre-reporter mice ideally suited for lineage tracing studies. Eur J Immunol (2007) 37:43-53. doi:10.1002/eji.200636745

85. Manfra DJ, Chen SC, Yang TY, Sullivan L, Wiekowski MT, Abbondanzo S, et al. Leukocytes expressing green fluorescent protein as novel reagents for 
adoptive cell transfer and bone marrow transplantation studies. Am J Pathol (2001) 158:41-7. doi:10.1016/S0002-9440(10)63942-9

86. Hasenberg A, Hasenberg M, Männ L, Neumann F, Borkenstein L, Stecher M, et al. Catchup: a mouse model for imaging-based tracking and modulation of neutrophil granulocytes. Nat Methods (2015) 12:445-52. doi:10.1038/ nmeth. 3322

87. Sasmono RT, Oceandy D, Pollard JW, Tong W, Pavli P, Wainwright BJ, et al. A macrophage colony-stimulating factor receptor-green fluorescent protein transgene is expressed throughout the mononuclear phagocyte system of the mouse. Blood (2003) 101:1155-63. doi:10.1182/blood-2002-02-0569

88. Swirski FK, Berger CR, Figueiredo JL, Mempel TR, von Andrian UH, Pittet $\mathrm{MJ}$, et al. A near-infrared cell tracker reagent for multiscopic in vivo imaging and quantification of leukocyte immune responses. PLoS One (2007) 2:e1075. doi:10.1371/journal.pone.0001075

89. Appaix F, Girod S, Boisseau S, Römer J, Vial JC, Albrieux M, et al. Specific in vivo staining of astrocytes in the whole brain after intravenous injection of sulforhodamine dyes. PLoS One (2012) 7:e35169. doi:10.1371/journal.pone.0035169

90. Suzuki R, Watanabe J, Arata S, Funahashi H, Kikuyama S, Shioda S. A transgenic mouse model for the detailed morphological study of astrocytes. Neurosci Res (2003) 47:451-4. doi:10.1016/j.neures.2003.08.008

91. Takano T, Han X, Deane R, Zlokovic B, Nedergaard M. Two-photon imaging of astrocytic $\mathrm{Ca} 2+$ signaling and the microvasculature in experimental mice models of Alzheimer's disease. Ann N Y Acad Sci (2007) 1097:40-50. doi:10.1196/annals.1379.004

92. Aoshi T, Zinselmeyer BH, Konjufca V, Lynch JN, Zhang X, Koide Y, et al. Bacterial entry to the splenic white pulp initiates antigen presentation to CD8+ T cells. Immunity (2008) 29:476-86. doi:10.1016/j. immuni.2008.06.013

93. Bullen A, Friedman RS, Krummel MF. Two-photon imaging of the immune system: a custom technology platform for high-speed, multicolor tissue imaging of immune responses. Curr Top Microbiol Immunol (2009) 334:1-29. doi:10.1007/978-3-540-93864-4_1

94. Tadokoro CE, Shakhar G, Shen S, Ding Y, Lino AC, Maraver A, et al. Regulatory $\mathrm{T}$ cells inhibit stable contacts between CD4+ T cells and dendritic cells in vivo. J Exp Med (2006) 203:505-11. doi:10.1084/ jem.20050783

95. Wei SH, Parker I, Miller MJ, Cahalan MD. A stochastic view of lymphocyte motility and trafficking within the lymph node. Immunol Rev (2003) 195:136-59. doi:10.1034/j.1600-065X.2003.00076.x

96. Siffrin V, Brandt AU, Radbruch H, Herz J, Boldakowa N, Leuenberger $\mathrm{T}$, et al. Differential immune cell dynamics in the CNS cause CD4+ T cell compartmentalization. Brain (2009) 132:1247-58. doi:10.1093/ brain/awn 354
97. Graham DB, Zinselmeyer BH, Mascarenhas F, Delgado R, Miller MJ, Swat W. ITAM signaling by Vav family Rho guanine nucleotide exchange factors regulates interstitial transit rates of neutrophils in vivo. PLoS One (2009) 4:e4652. doi:10.1371/journal.pone.0004652

98. Lämmermann T, Afonso PV, Angermann BR, Wang JM, Kastenmüller W, Parent CA, et al. Neutrophil swarms require LTB4 and integrins at sites of cell death in vivo. Nature (2013) 498:371-5. doi:10.1038/nature12175

99. McDonald B, Pittman K, Menezes GB, Hirota SA, Slaba I, Waterhouse CC, et al. Intravascular danger signals guide neutrophils to sites of sterile inflammation. Science (2010) 330:362-6. doi:10.1126/science.1195491

100. Hanson KM, Clegg RM. Two-photon fluorescence imaging and reactive oxygen species detection within the epidermis. Methods Mol Biol (2005) 289:413-22.

101. Wang X, Fang H, Huang Z, Shang W, Hou T, Cheng A, et al. Imaging ROS signaling in cells and animals. J Mol Med (Berl) (2013) 91:917-27. doi:10.1007/ s00109-013-1067-4

102. Andermann ML, Gilfoy NB, Goldey GJ, Sachdev RN, Wölfel M, McCormick DA, et al. Chronic cellular imaging of entire cortical columns in awake mice using microprisms. Neuron (2013) 80:900-13. doi:10.1016/j. neuron.2013.07.052

103. Wang BG, König K, Halbhuber KJ. Two-photon microscopy of deep intravital tissues and its merits in clinical research. J Microsc (2010) 238:1-20. doi:10.1111/j.1365-2818.2009.03330.x

104. Barretto RP, Ko TH, Jung JC, Wang TJ, Capps G, Waters AC, et al. Timelapse imaging of disease progression in deep brain areas using fluorescence microendoscopy. Nat Med (2011) 17:223-8. doi:10.1038/nm.2292

105. Kawakami R, Sawada K, Kusama Y, Fang YC, Kanazawa S, Kozawa Y, et al. In vivo two-photon imaging of mouse hippocampal neurons in dentate gyrus using a light source based on a high-peak power gain-switched laser diode. Biomed Opt Express (2015) 6:891-901. doi:10.1364/BOE.6.000891

106. Panatier A, Arizono M, Nägerl UV. Dissecting tripartite synapses with STED microscopy. Philos Trans R Soc Lond B Biol Sci (2014) 369:20130597. doi:10.1098/rstb.2013.0597

Conflict of Interest Statement: The authors declare that the research was conducted in the absence of any commercial or financial relationships that could be construed as a potential conflict of interest.

Copyright $\odot 2016$ Pietronigro, Zenaro and Constantin. This is an open-access article distributed under the terms of the Creative Commons Attribution License (CC BY). The use, distribution or reproduction in other forums is permitted, provided the original author(s) or licensor are credited and that the original publication in this journal is cited, in accordance with accepted academic practice. No use, distribution or reproduction is permitted which does not comply with these terms. 